\title{
Complex Nanotwin Substructure of an Asymmetric 59 Tilt Grain Boundary in a Silicon Polycrystal
}

\author{
A. Stoffers, ${ }^{1,}$ B. Ziebarth ${ }^{2,3}$ J. Barthel, ${ }^{4,5}$ O. Cojocaru-Mirédin, ${ }^{1}$ C. Elsässer, ${ }^{2}$ and D. Raabe ${ }^{1}$ \\ ${ }^{1}$ Max-Planck-Institut für Eisenforschung GmbH, Max-Planck-Straße 1, 40237 Düsseldorf, Germany \\ ${ }^{2}$ Fraunhofer-Institut für Werkstoffmechanik IWM, Wöhlerstraße 11, 79108 Freiburg, Germany \\ ${ }^{3}$ Karlsruher Institut für Technologie, Institut für Angewandte Materialien (IAM-CMS), \\ Engelbert-Arnold-Straße 4, 76131 Karlsruhe, Germany \\ ${ }^{4}$ Central Facility for Electron Microscopy, RWTH Aachen University, 52074 Aachen, Germany \\ ${ }^{5}$ Ernst Ruska-Centre for Microscopy and Spectroscopy with Electrons, \\ Forschungszentrum Jülich GmbH, 52425 Jülich, Germany
}

(Received 3 July 2015; published 2 December 2015)

\begin{abstract}
Grain boundaries in materials have substantial influences on device properties, for instance on mechanical stability or electronic minority carrier lifetime in multicrystalline silicon solar cells. This applies especially to asymmetric, less ordered or faceted interface portions. Here, we present the complex atomic interface structure of an asymmetric $\Sigma 9$ tilt grain boundary in silicon, observed by high resolution scanning transmission electron microscopy (HR-STEM) and explained by atomistic modeling and computer simulation. Structural optimization of interface models for the asymmetric $\Sigma 9$ and related symmetrical $\Sigma 9$ and $\Sigma 3$ tilt grain boundaries, by means of molecular-statics simulations with empirical silicon potentials in combination with first-principles calculations, results in a faceted asymmetric interface structure, whose grain-boundary energy is so low that it is likely to exist. The simulated local atomic structures match the observed HR-STEM images very well.
\end{abstract}

DOI: 10.1103/PhysRevLett.115.235502

PACS numbers: 61.72.Mm, 68.37.Ma, 88.40.jj

Multicrystalline silicon (mc-Si) is the most commonly used bulk material for solar cells. The presence of grain boundaries (GBs) in its microstructure profoundly influences the spatial distribution of impurity or dopant elements as well as the electronic characteristics of the material by increasing the recombination activity. For better understanding and manipulating these processes with the aim to enhance cell efficiency, it is required to reveal the detailed atomic structures of GBs, specifically of the less ordered ones. The majority of the GBs in mc-Si have relatively high coherence, which is described by a low- $\Sigma$ value of the associated coincidentsite lattice (CSL). The CSL concept quantifies the fraction of coinciding sites between the lattices of two differently orientated grains in contact. It serves as a measure of their crystallographic interface coherence. The $\Sigma$ value is the reciprocal of the ratio of coincidence lattice sites to the total number of lattice sites; i.e., high match is described by low $\Sigma$ values. This assumption implies although that the actual GB plane contains a high area density of such coincident lattice positions, which would explain a low GB energy and low solubility for dopants. However, using the $\Sigma$ value alone for deriving GB properties can lead to false conclusions since the CSL concept covers only 3 of the 5 macroscopic geometric degrees of freedom (DOF), neglecting variations of the GB plane (2 DOF), mutual rigid-body translations of the two grains (3 microscopic geometric DOFs), and of local atomic relaxations [1-3] (3N-6 microscopic atomic DOF for $N$ interface atoms).
The atomic structure of perfect twin boundaries (TBs), such as a coherent and symmetric $\Sigma 3(111)$ GB, has been described in the literature [4-6]. The first studies on the structure of higher- $\Sigma$ TBs were reported in the 1980s, such as for the symmetric $\Sigma 9(122)$ TB $[7,8]$. However, asymmetric higher- $\Sigma$ CSL GBs in Si have not been described in detail in the literature, although such nonideal GBs occur frequently in real materials with profound influence on the overall material response.

Here, we investigate the atomic structure of an asymmetric $\Sigma 9$ GB. High-resolution hardware aberration corrected scanning transmission electron microscopy (HR-STEM) enables us to distinguish individual atomic column positions having projected distances even below $1 \AA[9,10]$. Based on HRSTEM images taken of the $\Sigma 9$ GB plane along the [110] zone axis of the two abutting crystals we render a corresponding preliminary atomistic structure model. This model is refined using atomistic molecular-statics simulations based on empirical potentials and compared to the experimental observations. The calculations provide insight into the reconstruction mechanism of Si bonds at the GB which cannot be extracted directly from the HR-STEM images. Furthermore, GB energies are calculated using empirical potentials and first-principles methods in order to elucidate their stability. The calculated GB energies are compared with those of other stable, low- $\Sigma$ GBs from the literature.

The HR-STEM high-angle annular dark-field (HAADF) images were recorded with an FEI Titan 80-200 electron 
microscope operated at $200 \mathrm{kV}$. The microscope is equipped with a hexapole corrector to compensate for the strong spherical aberration of the probe-forming lens system. The residual aberrations up to the 3rd order have been corrected within an illumination aperture of $25 \mathrm{mrad}$. The projection system was adjusted such that the annular dark-field detector records the diffracted beams in the highangle regime of 90-200 mrad.

The specimen was taken from a mc-Si ingot grown by directional solidification. A thin TEM lamella was prepared by a site-specific procedure [11] using an FEI Helios Nanolab 600 dual beam focused ion beam (FIB) utilizing a custom-designed sample retainer [12]. The same instrument was used for precharacterization by electron backscatter diffraction (EBSD) to localize suitable sample regions with a common orientation of the neighboring grains, which is necessary for the subsequent HR-STEM analysis. Furthermore, the sample area was precharacterized by an electron beam induced current (EBIC) measurement at room temperature on a Zeiss EVO 40 SEM equipped with a Gatan EBIC system to analyze the minority carrier lifetime at the GBs. The lamella was extracted from an arrangement of three GBs, two $\Sigma 3$ and one $\Sigma 9 \mathrm{~GB}$, abutting at a triple junction, Fig. 1. The three grains are oriented with the [110] zone axis parallel to the electron beam and all GBs are oriented "edge on."

During specimen preparation the $\Sigma 9 \mathrm{~GB}$ appeared to be completely straight in the EBIC/EBSD image in Fig. 1(a) owing to its limited orientation imaging resolution ( $\sim 300 \mathrm{~nm}$ in the present case), but already in the STEM overview displayed in Fig. 1(b) we observe that the GB consists of at least two segments. The smaller segment lies in the direct vicinity of the triple junction. The atomic structure of this segment is visible in the detailed HRSTEM image of the triple junction in Fig. 1(c). It has the well-known structure of a symmetric $\Sigma 9$ (122) GB [13-15].
The distortions of the atomic arrangements are very small. For example, every single Si dumbbell in the HR-STEM image can be assigned to one of the two grains according to its orientation. The same HR-STEM image also shows the perfect symmetry of the adjacent $\Sigma 3(111)$ TBs. In view of this perfect symmetry it is not surprising that both $\Sigma 3$ GBs appear to be electrically inactive in terms of the EBIC signal observed in Fig. 1(a). In contrast, the $\Sigma 9$ GB shows a strong EBIC signal of $17.3 \%$, at least for the longer and asymmetric portion.

The change of the GB plane inclination occurring approximately $200 \mathrm{~nm}$ away from the triple point can be explained by the presence of the triple junction. In the immediate vicinity of the triple point, the $\Sigma 9(122) \mathrm{GB}$ plane fulfills the force equilibrium requirements together with the two $\Sigma 3(111)$ GBs. In greater distance from the triple junction, these boundary conditions are relaxed; i.e., the interface is free to change, realizing a complex asymmetric structure at a (111)|(115) interface in the present case. Contrary to the symmetric $\Sigma 9$ (122) segment, the atomic structure of the asymmetric segment shows strong distortions, but nevertheless it repeats periodically with almost perfect accuracy over a range of several $100 \mathrm{~nm}$. The structure has a period length of $2 \mathrm{~nm}$ along the GB, as illustrated in Fig. 2. At the interface we identify $10 \mathrm{Si}$ dumbbells, which cannot be assigned to one of the two grains, as they have a completely different orientation (marked blue). Furthermore, there are four apparently single-atomic columns per period (marked yellow). The distorted dumbbells are oriented such that they resemble the structure of several short stacking faults or "nano$\Sigma 3$ TBs," consisting of only 4 to 8 atoms. Within each period of the complex GB structure, the HAADF signal drops significantly. However, the intensity peaks due to the projected atomic columns can still be recognized in the image. The reduction of the HAADF intensity can be due to
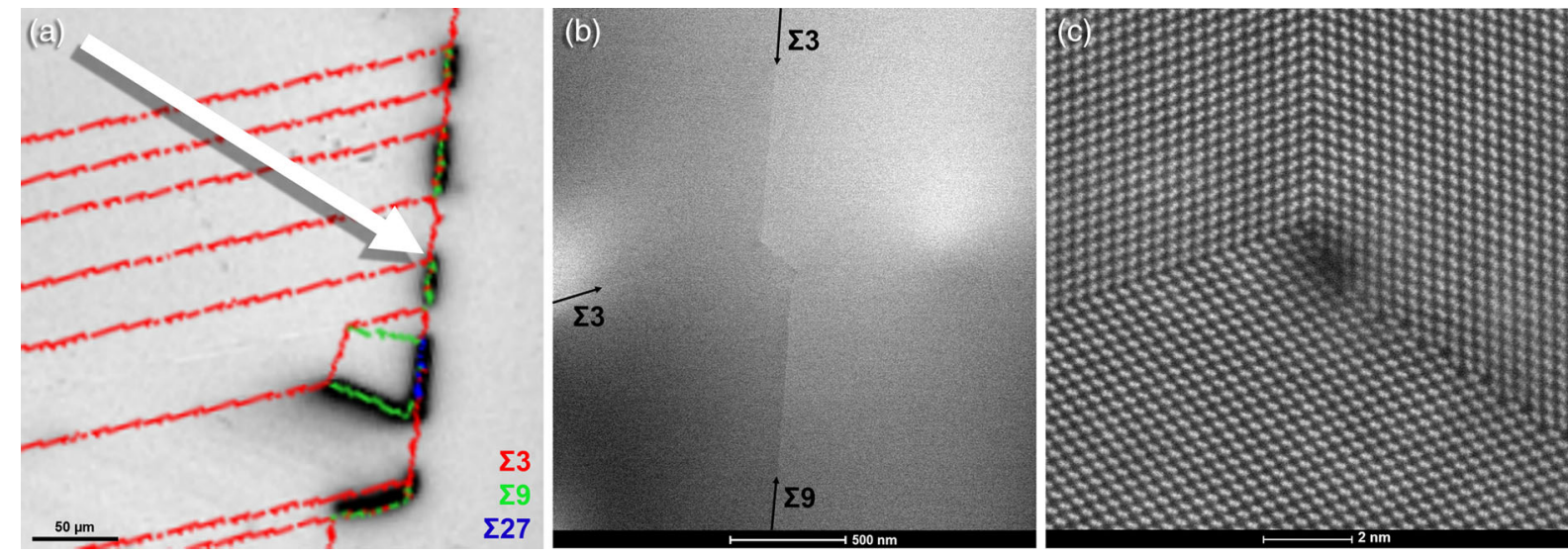

FIG. 1 (color online). (a) EBIC map (b/w) of the studied sample area in correlation with the CSL character obtained by EBSD (RGB). The arrow indicates the analyzed triple junction. (b) Overview of the TEM specimen containing a triple junction of two $\Sigma 3$ and one $\Sigma 9$ GBs. The $\Sigma 9$ GB changes its interface orientation approximately $200 \mathrm{~nm}$ away from the triple point. (c) High-resolution HAADF STEM image of the triple junction. All three GBs meeting at the triple junction have a symmetric structure. 


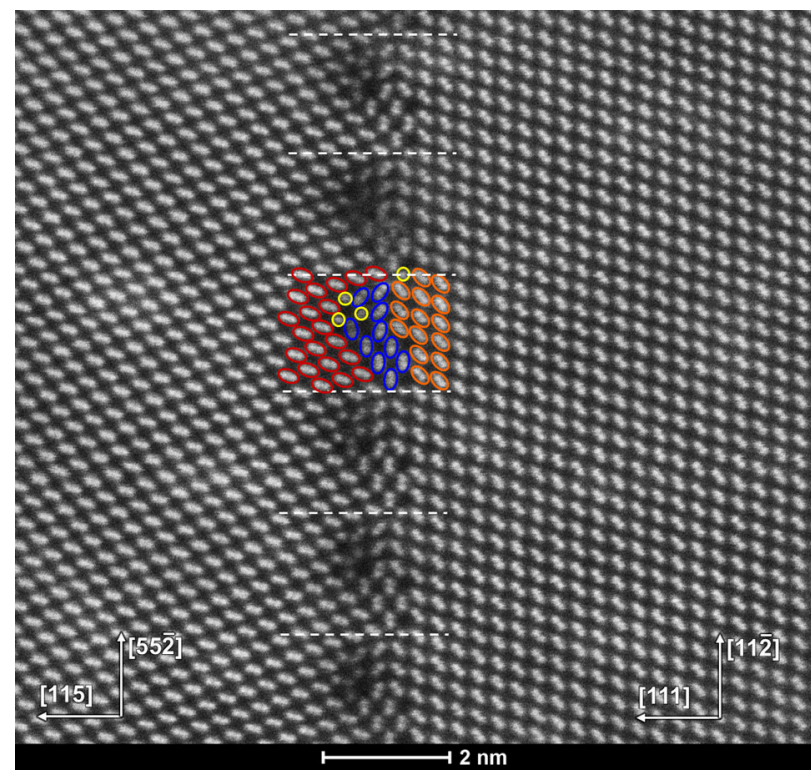

FIG. 2 (color online). HR-STEM image of an asymmetric $\Sigma 9(111 \mid 115)$ boundary of two Si grains in the [110] projection. The period of the complex GB structure is indicated by white dashed lines. Dumbbell columns belonging to the left grain are marked by red ellipses, while those belonging to the right grain are marked orange. The dumbbells marked by blue ellipses deviate in their orientation and cannot be assigned to one of the grains. Four single Si atom columns are marked by yellow circles.

geometrical distortions of the crystal lattice as discussed by Couillard et al. [13], as well as due to chemical defects such as vacancies or the presence of impurity atoms at the GB.

Starting from the experimental HR-STEM images, a preliminary atomistic model of the asymmetric GB was created by placing $\mathrm{Si}$ atoms at the intensity-peak positions. Free surfaces to a vacuum volume were introduced in the structure model to terminate the two grains in the relaxation calculations. The orthorhombic supercell with periodic boundary conditions is displayed in Fig. 3(a). It contains a slab of $360 \mathrm{Si}$ atoms and extends over $19.95 \AA$ along the interface, over $56.96 \AA$ perpendicular to the interface, and over $7.679 \AA$ along the viewing direction.

Classical atomistic molecular-statics simulations using empirical interatomic potentials, namely, Tersoff [17], Stillinger-Weber [18], and EDIP [19] potentials, have been used to relax the interface structure and to calculate the GB energy. Figure 3 displays the relaxed structure along two directions. Figure 4(a) shows an overlay of the relaxed structure with the experimental image, demonstrating qualitatively a good match between experiment and calculation. However, the structure model does not describe the lower HAADF intensity observed for some atomic columns in the GB region. Such a decrease of the HAADF intensity may occur due to several reasons: reduced local object thickness, chemical variation, atomic site vacancies, and/or static atomic displacements. High-resolution EDX analysis of the sample composition at the GB did not

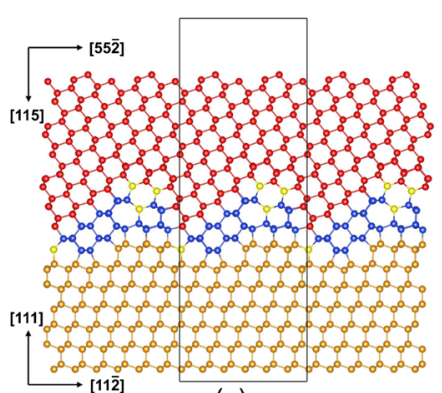

(a)

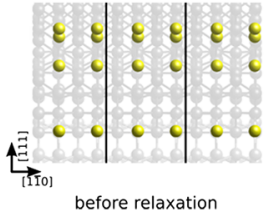

FIG. 3 (color online). (a) Relaxed atomic structure model of the faceted $\Sigma 9(111 \mid 115) \mathrm{GB}$ viewed along the [110] direction. Red spheres and orange spheres depict the two different grains. The facet is colored in blue, while yellow balls represent single atom columns. The rectangular box marks the borders of the supercell used in the calculations. The supercell extends over $19.95 \AA$ along the interface, over $56.96 \AA$ perpendicular to the interface, and over $7.679 \AA$ along the viewing direction, containing two free surfaces at the top and at the bottom. The structure image was created by the program VESTA [16]. (b) Section of the atomic structure model in Fig. 3 viewed along the [112] direction before and after relaxation by empirical potentials. The relaxation results in a bond reconstruction of atoms from the single atom columns (yellow) due to displacements along the [110] direction. The reconstruction pattern between the different single atom columns is not limited to the two shown configurations.

indicate the presence of chemical species other than $\mathrm{Si}$. We also assume that strong variations of the object thickness are rather unlikely on such a small length scale. We therefore investigated the initial structure and two structure modifications by STEM image simulations in order to reproduce the reduced intensity at the GB, Figs. 4(b)-4(d). The image simulations were carried out using the Dr. Probe software [20] in conjunction with parameters close to the experimental conditions. The electron probe aberrations are assumed to be corrected within the illumination aperture of $25 \mathrm{mrad}$, providing a diffraction limited probe size of $1 \AA$ (FWHM). The same probe diameter was used to describe the incoherent resolution limitation by the geometric profile of the electron source. The image intensity was extracted from the diffracted beams within a high-angle annular detection range of 80 up to $200 \mathrm{mrad}$. In the first modification we reduced the atomic site occupancies at image locations appearing darker in the experiment to values between 0.7 and 0.9 . In the second modification we introduced random but static atomic displacements with a maximum rms displacement amplitude of $30 \mathrm{pm}$ at the atomic sites with the lowest observed HAADF intensity. The modifications were realized in a large supercell of 


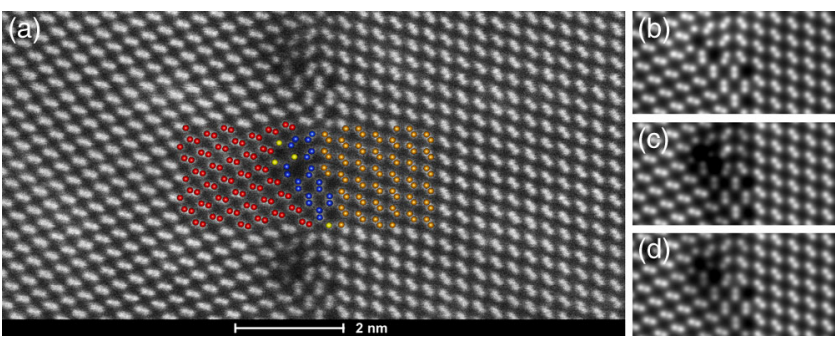

FIG. 4 (color online). (a) Overlay of experimental HR-STEM image with the colored projection of the relaxed structure model. (b) Simulated image of the proposed structure at $12 \mathrm{~nm}$ thickness showing all atom columns with similar intensity. (c) Simulated image of the proposed structure with partial site occupancies at the interface and (d) with static atomic displacements at the interface. The image simulations were carried out with the Dr. Probe software package [20].

$12 \mathrm{~nm}$ thickness. While the simulated image of the initial structure in Fig. 4(b) does not show any intensity fluctuations of the atomic columns, it still reproduces almost perfectly the location of the intensity peaks observed in experiment. We therefore conclude that the atomic positions as obtained in the investigated projection are correct. Both structure modification scenarios, partial site occupancies in Fig. 4(c), and static atomic displacements in Fig. 4(d), reproduce the reduced peak intensities for the modified columns at the grain boundary in the simulated STEM images, appearing very similar to the experimental image. Both structure modifications applied in the simulations can indeed occur in real material, such as static displacements due to local strains or $\mathrm{Si}$ substitution by light elements, e.g., by carbon, which would be difficult to detect by EDX when occurring in low amounts. In conclusion, we can so far not decide which of the two effects actually applies or dominates in the real material.

From the classical atomistic molecular-statics simulation it turns out that the atoms in the apparently single-atomic columns are threefold coordinated in the nonrelaxed configuration, but can reconstruct to fourfold coordination by pairwise displacements along the [110] direction. From the HR-STEM image, this reconstruction is not unique and two possible patterns are shown in Fig. 3(b). Eight possible reconstruction patterns exist within the used supercell, which is highlighted by the solid black frame in Figs. 3(a) and 3(b).

To study the thermodynamic stability of the GB and its dependence on the various reconstruction patterns of the single atomic columns, GB energies have been calculated according to

$$
\gamma_{\mathrm{GB}}=\frac{\mathrm{E}_{\mathrm{GB}}-\mathrm{N}_{\mathrm{Si}} \cdot \mu_{\mathrm{Si}}}{\mathrm{A}_{\mathrm{GB}}}-\gamma_{\mathrm{SurfI}}-\gamma_{\mathrm{SurfII}}
$$

where $E_{\mathrm{GB}}$ is the total energy of the GB supercell, $N_{\mathrm{Si}}$ is the number of $\mathrm{Si}$ atoms, $\mu_{\mathrm{Si}}$ is the chemical potential of $\mathrm{Si}$ in the equilibrium diamond structure, and $A_{\mathrm{GB}}$ is the interface area of the GB. $\gamma_{\text {SurfI }}$ and $\gamma_{\text {SurfII }}$ are the surface energies of the two free surfaces in the supercell, namely, (111) and (115) surfaces. It is known that empirical potentials yield correct GB structures, but tend to overestimate interface energies [21-23]. In order to elucidate this deficit, interface energies have been calculated for a set of different GBs and subsequently related to $a b$ initio density functional theory (DFT) results which have been previously obtained [24]. The results shown in Fig. 5 indicate that all empirical potentials give similar energies for all considered GBs. The empirical potentials capture the correct trend of the GB energies but overestimate their values by a factor of 2-3 compared to the GB energies obtained from the DFT calculations. Insignificant changes of the GB energies between 0.71 and $0.75 \mathrm{~J} / \mathrm{m}^{2}$ are found for the different reconstruction patterns of the yellow single-atom columns. The GB energy of the asymmetric $\Sigma 9$ GB has been obtained from a static DFT calculation based on the structure that was previously relaxed by empirical potentials, but the volume of the structure has been rescaled to that of the DFT equilibrium value. Its energy is found to be in the range of the highly symmetric low- $\Sigma$ GBs. This explains its stability over a large spatial region of more than $100 \mathrm{~nm}$. The relaxation of the GB structure into a sequence of short $\Sigma 3$ nano-TB segments could be one reason for the surprisingly low energy values found for this complex GB structure.

It has been shown in previous studies that the GB plane and symmetry have a strong influence on the impurity segregation and on the recombination activity in mc-Si. For example, Kojima and co-workers [25] recently showed increased recombination activity and nickel segregation for

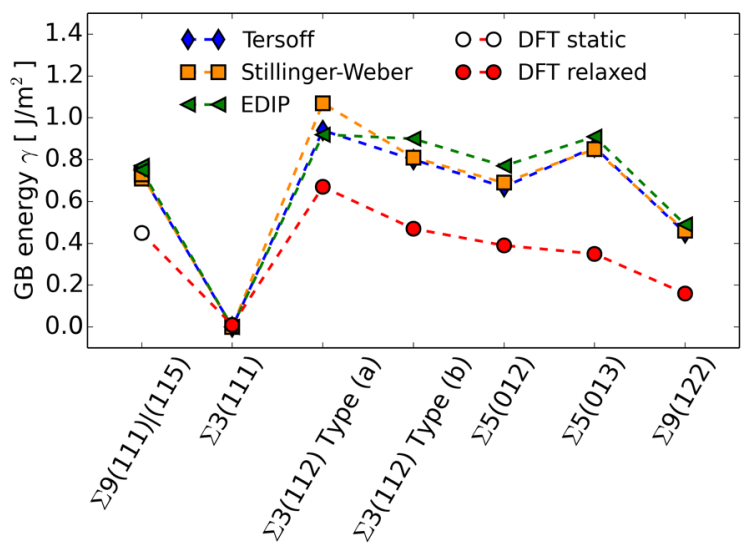

FIG. 5 (color online). Calculated GB energies for various GBs. DFT values are taken from Ref. [24]. The DFT value of the GB energy for the asymmetric $\Sigma 9(111) \mid(115)$ has been obtained from a static calculation using the relaxed structure as obtained by empirical potentials. The volume of that structure has been rescaled to that of the DFT equilibrium value. The $23(112)$ GB types termed as (a) and (b) correspond to the symmetric and asymmetric इ3(112) GBs, respectively, in Ref. [24]. 
curved and asymmetric $\Sigma 9$ GBs by EBIC and synchrotronbased x-ray analysis. Similar results were obtained for the case of interstitial $\mathrm{Fe}$ impurity segregation at different types of $\Sigma 3$ GBs by Chen et al. [26], which has also been validated by DFT calculations [24]. Another example is given by our preceding correlative analysis of EBSD, EBIC, and atom probe tomography measurements [27]. The asymmetric $\Sigma 9$ GB studied here also shows a strong EBIC contrast of $17.3 \%$, as shown in Fig. 1(a), which is detrimental to the solar cell efficiency. This can be either due to additional energy states, impurity segregation, or a combination of the two. Both reasons for a strong EBIC contrast are supported by the complex atomic GB structure, in particular, by the above-mentioned reasons for the periodic reduction of the HAADF signal observed along the GB, partial site occupancies, and static atomic displacements. Also, in other material systems, asymmetric and faceted GBs are known to have a strong influence on the local material properties such as recently shown by $\mathrm{Li}$ et al. for a faceted $\Sigma 9 \mathrm{~GB}$ in CdTe [28]. These observations indicate that such types of defects occur rather frequently and are not an exception, demanding in turn further studies contributing a better fundamental understanding of the corresponding structural properties at GBs.

In conclusion, we studied the atomic structure of an asymmetric $\Sigma 9$ GB related to a triple-point arrangement of $\Sigma 3$ and $\Sigma 9$ GBs in mc-Si by HR-STEM. We provide direct evidence that the orientation of the GB plane has a strong impact on the atomic interface structure. The change in the GB plane inclination is explained in terms of the topology constraints imposed by the triple junction. In greater distance from the triple junction, the $\Sigma 9 \mathrm{~GB}$ plane assumes an asymmetric structure at a (111)|(115) interface extending over several $100 \mathrm{~nm}$ in the present case. Surprisingly, the interface structure is built of short segments of stacking faults or $\Sigma 3 \mathrm{TBs}$, respectively. The existence of such energetically favorable substructural motifs explains the high stability of this asymmetric GB and the surprisingly low GB energy found in the molecular statics calculations. In addition to the high thermodynamic stability and structural periodicity of the asymmetric GB, EBIC measurements document its enhanced recombination activity relative to the symmetric GBs. Topological constraints require the presence of such asymmetric GBs in polycrystals. Hence, a better understanding of their atomic structure enables the development of new passivation techniques and, consequently, of solar cells with higher efficiency.

The authors would like to thank Dr. Winfried Seifert for providing the mc-Si sample and for performing the EBIC analysis. Part of this work was financed by the Federal Ministry of Education and Research (BMBF 03X5522A), by the German Research Foundation (MA 1280/40-1), and by the Hans L. Merkle foundation of the Robert Bosch GmbH.
* Corresponding author. stoffers@mpie.de

[1] W. Bollmann, Crystal Defects and Crystalline Interfaces (Springer, New York, 1970).

[2] A. P. Sutton and R. W. Balluffi, Interfaces in Crystalline Materials, Monographs on the Physics and Chemistry of Materials (Clarendon Press; Oxford University Press, Oxford New York, 1995), p. 51.

[3] D. Wolf, Atomic-Level Geometry of Crystalline Interfaces (Chapman and Hall, London, 1992).

[4] O. L. Krivanek, S. Isoda, and K. Kobayashi, Philos. Mag. 36, 931 (1977).

[5] J. Narayan and A. S. Nandedkar, Philos. Mag. B 63, 1181 (1991).

[6] Y. Zhang, H. Ichinose, K. Ito, and Y. Ishida, J. Electron Microsc. 48, 245 (1999).

[7] C. Danterroches and A. Bourret, Philos. Mag. A 49, 783 (1984).

[8] J. Thibault, J. L. Putaux, A. Jacques, A. George, H. M. Michaud, and X. Baillin, Mater. Sci. Eng. 164, 93 (1993).

[9] N. Dellby, O. L. Krivanek, P. D. Nellist, P. E. Batson, and A. R. Lupini, J. Electron Microsc. 50, 177 (2001).

[10] O. Krivanek, P. Nellist, N. Dellby, M. Murfitt, and Z. Szilagyi, Ultramicroscopy 96, 229 (2003).

[11] R. Langford, Y. Huang, S. Lozano-Perez, J. Titchmarsh, and A. Petford-Long, J. Vac. Sci. Technol. B 19, 755 (2001).

[12] M. Herbig, P. Choi, and D. Raabe, Ultramicroscopy 153, 32 (2015).

[13] M. Couillard, G. Radtke, and G. A. Botton, Philos. Mag. 93, 1250 (2013).

[14] J. Putaux and J. Thibault-Dessaux, J. Phys. Colloq. 51, C1-323 (1990).

[15] M. Elkajbaji and J. Thibault-Desseaux, Philos. Mag. A 58, 325 (1988).

[16] K. Momma and F. Izumi, J. Appl. Crystallogr. 44, 1272 (2011).

[17] J. Tersoff, Phys. Rev. B 38, 9902 (1988).

[18] F. H. Stillinger and T. A. Weber, Phys. Rev. B 31, 5262 (1985).

[19] J. F. Justo, M. Z. Bazant, E. Kaxiras, V. V. Bulatov, and S. Yip, Phys. Rev. B 58, 2539 (1998).

[20] J. Barthel, http://www.er-c.org/barthel/drprobe/ (2015).

[21] P. Keblinski, D. Wolf, S. Phillpot, and H. Gleiter, J. Mater. Res. 13, 2077 (1998).

[22] V. Y. Lazebnykh, and A. S. Mysovsky, arXiv:1308.3802.

[23] S. von Alfthan, P. D. Haynes, K. Kaski, and A. P. Sutton, Phys. Rev. Lett. 96, 055505 (2006).

[24] B. Ziebarth, M. Mrovec, C. Elsässer, and P. Gumbsch, Phys. Rev. B 91, 035309 (2015).

[25] T. Kojima, T. Tachibana, N. Kojima, Y. Ohshita, K. Arafune, A. Ogura, and M. Yamaguchi, Jpn. J. Appl. Phys. 53, 04ER20 (2014).

[26] B. Chen, J. Chen, T. Sekiguchi, M. Saito, and K. Kimoto, J. Appl. Phys. 105, 113502 (2009).

[27] A. Stoffers, O. Cojocaru-Mirédin, W. Seifert, S. Zaefferer, S. Riepe, and D. Raabe, Prog. Photovoltaics: Res. Appl., doi: 10.1002/pip.2614 (2015).

[28] C. Li et al., Phys. Rev. Lett. 112, 156103 (2014). 